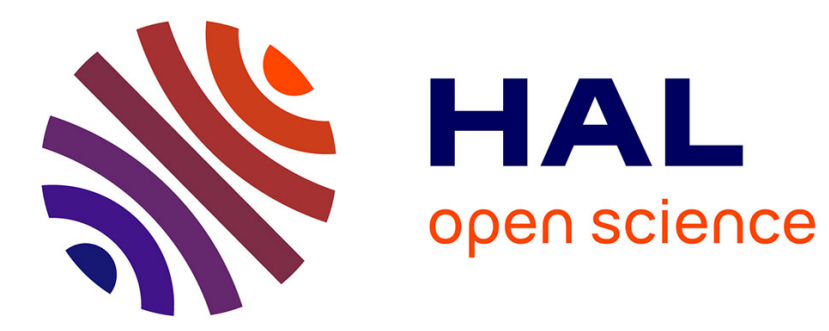

\title{
Pour une archéologie de la subvention agricole
}

Sylvain Brunier, Nicolas Krauberger

\section{To cite this version:}

Sylvain Brunier, Nicolas Krauberger. Pour une archéologie de la subvention agricole. Frédéric Chauvaud; Laurent Willemez. Justice et sociétés rurales du XVIe siècle à nos jours, pp.21-38, 2011, 9782753513556. hal-01439411

\section{HAL Id: hal-01439411 \\ https://hal.science/hal-01439411}

Submitted on 18 Jan 2017

HAL is a multi-disciplinary open access archive for the deposit and dissemination of scientific research documents, whether they are published or not. The documents may come from teaching and research institutions in France or abroad, or from public or private research centers.
L'archive ouverte pluridisciplinaire HAL, est destinée au dépôt et à la diffusion de documents scientifiques de niveau recherche, publiés ou non, émanant des établissements d'enseignement et de recherche français ou étrangers, des laboratoires publics ou privés. 


\title{
Pour une archéologie de la subvention agricole
}

\author{
Sylvain Brunier et Nicolas KraUberger
}

Ouvert au grand public depuis avril 2009, le site TelePAC offre à tout un chacun la possibilité de connaître le montant des aides européennes perçues par chaque exploitant agricole ${ }^{1}$. Mais TelePAC a été conçu avant tout pour les professionnels de l'agriculture française dans le cadre du développement de la politique de l'e-administration, dont l'objectif est de mettre en place un véritable guichet électronique de déclaration et de consultation des dossiers de demandes d'aides directes déposés auprès du ministère de l'Agriculture, de la Pêche et de l'Alimentation. En ligne depuis 2004, ce portail repose sur une base de données pensée depuis 1994 par la mission ministérielle responsable de la mise en ouvre du système intégré de gestion et de contrôle des aides communautaires (SIGC) pour la Politique Agricole Commune ${ }^{2}$. Ce SIGC combine trois bases de données: la première permet de mettre en rapport les différentes demandes des agriculteurs de manière à servir de support à des contrôles automatisés, la seconde cartographie les parcelles agricoles et sert de support aux déclarations de surfaces et aux contrôles du parcellaire, la troisième comptabilise les effectifs des animaux et autorise un contrôle de leur éligibilité.

Du point de vue administratif, cette innovation est considérée comme une simplification technique évitant d'encombrer les bureaux des directions Départementales de l'Agriculture et de la Forêt de milliers de formulaires papier ${ }^{3}$. De plus, la publication de renseignements nominatifs (nom, adresse, montant des aides attribuées) sur chaque bénéficiaire par-

1. Ministère de l'Agriculture, de la Pêche et de l'Alimentation, TelePAC, https://www1.telepac.agricul-

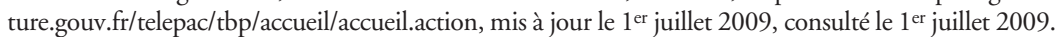

2. Anne Chausserie, Gabrielle-Angeline Cosson, Véronique Deaud, Me Bernard Peignot, Guide. Les aides à l'agriculture, Paris, Éd. Agridécisions, 1997, 189 p.

3. Telepac, Réalisation: Mathieu PERDONCIN, Dijon, Educagri, 2008, DVD (36 min 16 s.), son, coul. 
ticipe du souci d'améliorer la transparence dans l'attribution des fonds publics européens dévolus à l'agriculture. Objectivée à partir de TelePAC, la subvention agricole n'apparaît plus comme un simple don, mais comme une pratique mettant en circulation de l'argent et des informations. Tout se passe alors comme si ces échanges étaient réglés par les différences de potentiel entre deux exigences de justice de natures différentes: la subvention agricole est une aide destinée à compenser les inégalités socio-spatiales qui pénalisent les agriculteurs à l'intérieur du territoire européen (parité de revenu avec l'ensemble de la population non agricole) et sur le marché économique globalisé (compétitivité des prix à l'exportation); elle est aussi un subside public dont la juste répartition légitime la recherche d'une efficacité administrative toujours plus grande.

Si on considère TelePAC comme un système qui allie des procédures et des effets de connaissance (renseigner des formulaires) à une série de mécanismes particuliers susceptibles d'induire des comportements ou des discours (devenir un ayant-droit), alors le travail de l'historien consiste à examiner comment s'est constitué ce nœud de savoir-pouvoir dans le temps ${ }^{4}$. Dédommager les pertes des sinistrés après calamités agricoles, reconstruire les fermes détruites par la guerre, soutenir les régions défavorisées sur le plan de la productivité: ces pratiques mettent en jeu différents types de subventions agricoles qui pourraient être inscrites rétrospectivement dans un processus historique continu de modernisation rurale. Mais cette démarche laisserait échapper les singularités historiques propres à chaque situation, empêchant de saisir l'ambivalence de la notion de justice sans cesse reconfigurée par les échanges d'aides et d'informations entre l'administration et les sociétés rurales.

"L'inventaire des différences " permet de sortir de cette impasse et de se pencher sur les variations historiques de la légitimité de la subvention agricole, en la réinscrivant dans un large dispositif d'incitation-compensation, perceptible dans les Alpes depuis l'époque moderne ${ }^{5}$. Dans cette optique, l'étude de la répartition des dégrèvements fiscaux extraordinaires pour catastrophe naturelle dans la seconde moitié du XVIII siècle en Dauphiné et la ventilation des fonds de secours dans le dernier tiers du XIXe siècle en Isère permet de comparer deux manières d'indemniser les sinistrés après calamités agricoles. Au XXe siècle, les deux décennies qui suivent la Seconde Guerre Mondiale apparaissent particulièrement intéressantes. Dans cette période de rupture et de bouleversement plus que de transformation, deux initiatives se dégagent dans les Alpes du Nord françaises: la politique dite de reconstitution agricole à l'occasion de la reconstruction des villages détruits,

\footnotetext{
4. Michel Foucault, "Qu'est-ce que la critique? ", Conférence du 27 mai 1978 devant la Société française de philosophie in Bulletin de la Société française de philosophie, $\mathrm{n}^{\circ}$ 2, avril-juin 1990, p. 38.

5. Paul VeYNe, L'inventaire des différences: leçon inaugurale au Collège de France, Paris, Le Seuil, 1976, 61 p.
} 
et la politique de développement des territoires jugés défavorisés par le biais des zones-témoins au début des années 1950. Il s’agit de se demander si la généralisation progressive de l'utilisation de la subvention pour orienter les pratiques agricoles relève d'une simple extension des procédés décrits pour le XVIII e et le XIX ${ }^{e}$ siècle ou si on peut plutôt parler d'une intensification qui en modifierait la nature même. Une acception de la justice spécifique aux rapports entre administration et sociétés rurales doit alors prendre en compte une série d'agencements différentiels: entre compensation ponctuelle et incitation permanente, légitimité politique et légitimité administrative, droit et norme.

\section{Les dégrèvements fiscaux extraordinaires pour catastrophes naturelles en Dauphiné (1744-1785)}

Sous l'Ancien Régime, le poids économique que les catastrophes naturelles faisaient peser sur l'ensemble des sujets français a conduit la monarchie à développer un système d'indemnisation principalement articulé sur l'impôt. Une inondation, un tremblement de terre ou ouragan pouvaient alors être l'objet d'une demande de dégrèvement fiscal de la part d'une communauté rurale ou d'un particulier. Au cours du XVIII e siècle, les diverses procédures régionales d'indemnisation se stabilisèrent peu à peu autour de jurisprudences plus ou moins voisines ${ }^{6}$. Ainsi, en France, à l'époque moderne, il existait une voie de recours à la mansuétude royale pour tout cultivateur taillable ayant subi des pertes du fait d'une gelée, d'un incendie, d'une épizootie ou de ravines.

Dans un pays d'élection comme la province de Dauphiné, le système d'indemnisation comprenait deux volets. Depuis l'arrêt du 24 octobre 1639, le roi octroyait annuellement à la province un allègement ordinaire de $55.000 £^{7}$. La reconduction permanente de ce fonds de réserve visait à combler les difficultés inhérentes à la nature frontalière de cette province de montagne. Par ailleurs, chaque année, l'intendant pouvait solliciter auprès du roi un dégrèvement extraordinaire de taille dont le montant devait être justifié par la nécessité d'amortir des événements particuliers susceptibles de

\footnotetext{
6. Sur la grande différence opposant principalement pays d'État et pays d'élection, voir René FAVIER, "Une aide instrumentalisée. L'impôt et l'indemnisation des catastrophes naturelles dans la France du XVIII siècle" in Les modalités de paiement de l'État moderne. Adaptation et blocage d'un système comptable, Journée d'études du 3 décembre 2004 / éd. par Marie-Laure LEGAY, Paris, Comité pour l'histoire économique et financière de la France, 2007, p. 107-131.

7. René FAVIER, "La monarchie d'Ancien Régime et l'indemnisation des catastrophes naturelles à la fin du XVIII siècle: l'exemple du Dauphiné " in René FAVIER (dir.), Les pouvoirs publics face aux risques naturels dans l'histoire, Grenoble, MSH-Alpes, 2002, p. 71-104; René FAVIER, "From solidarity to individual compensation: assistance mechanisms faced with the emergence of liberalism in France during the 18th century " in René FAVIER, Christian PFISTER (dir.), Solidarité et assurance. Les sociétés européennes face aux catastrophes (17e-20e s.), Grenoble, MSH-Alpes, 2007, p. 57-79.
} 
mettre en péril la continuité des prélèvements fiscaux. C'est sous l'intendance de Fontanieu, durant la décennie 1720, que le calcul de ce volant spécial de l'aide se normalisa. Les sinistres devaient faire l'objet de procès-verbaux de déclaration par les officiers des communautés, puis être vérifiés et quantifiés sur le terrain par les subdélégués de l'intendant. Ce dernier se chargeait d'envoyer toutes ces pièces, associées à l'état des récoltes de l'année, au Conseil royal qui fixait le montant du dégrèvement extraordinaire s'additionnant au dégrèvement annuel ordinaire de l'année suivante. Ces fonds étaient ensuite ventilés aux communautés et particuliers plaignants par les officiers municipaux. Au cours de cet aller-retour, la clef de l'aboutissement des demandes d'indemnisation restait les réseaux de relations capables de porter les plaintes concernant des parcelles endommagées jusque sous la plume de l'intendant, à travers les rouages que représentaient les subdélégués de l'intendant chargés des enquêtes ex ante. Pour finir, le roi pouvait, certaines années, accorder en dernier recours des secours exceptionnels qui venaient s'ajouter à l'ensemble de ces dégrèvements ${ }^{8}$.

Dans les formulaires de demande standardisés et pré-imprimés que l'intendance mettait à disposition des sujets à partir des années 1760-1770, deux éléments étaient prépondérants pour la procédure d'indemnisation: la liste exhaustive des renseignements exigés lors de la constitution du dossier qui pouvait être individuel ou collectif, ainsi que les motifs au titre desquels on se faisait indemniser. Pour l'année 1785, tout taillable de l'élection de Grenoble devait justifier de son identité (nom, prénom, âge), de sa communauté de résidence, du montant des dommages et des motifs des pertes. Sur quatre cents six dossiers indemnisés, ces motifs peuvent être classés en six catégories (voir tableau des motif de dégrèvement - Annexe $1^{9}$ ).

La catégorie " dommage naturel » rassemble dans le tableau ci-dessus toutes les demandes qui firent suite à des événements climatiques ayant occasionné des dégâts matériels à des cultivateurs ${ }^{10} .62 \%$ des demandes concernaient ainsi l'activité agricole, mobilisant $41 \%$ du montant de l'aide extraordinaire attribuée à l'élection de Grenoble. En calculant les indemnisations moyennes, les "dommages naturels " représentent le second secteur aidé par l'État derrière les « travaux publics » (mutation du réseau

8. René FAVIER, op. cit., 2002, p. 77.

9. Archives départementales de l'Isère (AD38) 2C473-475: Taille. Dégrèvement. Extraits de l'état de distribution de la somme accordée par le roi en diminution de la taille en considération des pertes et dommages causés par les intempéries. Élection de Grenoble, 1785.

10. La normalisation de la procédure s'accompagna parallèlement d'une normalisation de la catégorie des sinistres sur lesquels pouvait porter les allègements fiscaux: « incendies, épizooties, gelées, grêles, ouragan, inondations, glissements de terrain, peste et gens de guerre ", AD38, 4E26 (Clavans), AA7: Ordonnance de l'intendant Fontanieu fixant les modalités de déclaration de sinistres, 20 octobre 1729. 
routier dans la province) ${ }^{11}$, attendu que les catégories " économiques » et " charité " recouvraient dans les faits des moyens détournés d'instrumentalisation des financements publics ${ }^{12}$.

Entre 1744 et 1758, les fonds distribués au titre de ces deux catégories problématiques furent au centre d'une discorde qui opposa violemment le nouvel intendant de La Porte aux officiers de la Chambre des comptes du Dauphiné. Le rapport de force qui s'engagea permet de mettre en lumière les deux logiques bien distinctes qui travaillaient alors le monopole de la manipulation légitime de ces aides. Ce que la Chambre des comptes reprochait principalement à l'Intendant, plus que des malversations dans la distribution des dégrèvements (faux motifs pour la rémunération des subdélégués), c'était surtout de n'en rendre aucun compte, «bail au rabais ny reception d'œuvre, de sorte que par cette voye la Chambre des Comptes se trouvoit hors d'état de pouvoir juger sainement si l'employ des deniers de Votre majesté avoit été fait conformément à sa destination 13 \%. Qu'une partie non négligeable de ces subsides royaux ait été déviée de l'indemnisation des sinistrés au profit de la consolidation de l'assise symbolique d'un personnage aussi central que l'Intendant dans la genèse de l'État moderne n'a rien d'extraordinaire. À la différence du XVIIe siècle ${ }^{14}$, au siècle des Lumières la gloire circulait sans entrave entre le roi et ses missi dominici ${ }^{15}$. Par ses choix arbitraires que motivait tout un faisceau de raisons pratiques convergeant vers la construction de sa position de premier administrateur de la province, de La Porte remettait en question la légitimité de la Chambre des comptes et son existence même. Dans la seule élection de Grenoble, il y eu pour l'année 1748, rappellent-elle au roi, "pour près de 12000 livres distribuées sous des noms dont on n'a pu reconnoitre aucun dans les rolles de tailles de lad. année qu’on a exactement vérifié dans les deux cent trente trois communautés dont ladite élection est composée ${ }^{16}$ ». Afin d'appuyer leurs remontrances, les officiers entreprirent de leur propre initiative d'élaborer une liste exhaustive des taillables des cinq élections qu'ils purent recouper avec les rôles de taille déjà existants ${ }^{17}$.

11. Bernard LEPETIT, Chemins de terre et voies d'eau: réseaux de transports et organisation de l'espace en France: 1740-1840, Paris, EHESS, 1984, 148 p.

12. René FAVIER, op. cit., 2002, p. 84-86.

13. $\mathrm{AD} 38,2 \mathrm{C} 504, \mathrm{n}^{\circ} 24$ : Très humbles et très respectueuses remontrances que présentes au Roy notre très honoré souverain seigneur les gens tenants la Chambre des comptes de Dauphiné, s.d.

14. Bernard BONNIN, René FAVIER, L'intendance de Dauphiné en 1698. Edition critique du mémoire rédigé par l'intendant Étienne-Jean Bouchu " pour l'instruction du Duc de Bourgogne ", Paris, CTHS, 2005, 220 p.

15. La responsabilité qui pesait sur la fonction d'intendant de pays d'élection en raison de ce mode particulier de distribution des aides avait d'ailleurs fait l'objet d'une mise en garde par l'un des premiers d'entre-eux: l'Intendant Bouchu en 1688, voir René Favier, op. cit., 2004, p. 113.

16. $\mathrm{AD} 38,2 \mathrm{C} 504, \mathrm{n}^{\circ} 11$ : Lettre du 20 décembre 1755.

17. AD38, 2C504, $\mathrm{n}^{\circ} 17,18,19$ : Extraits de listes nominatives par élection, s.d. 
En l'absence de tout moyen coercitif de contrôle de l'activité de l'intendant, la Chambre se retrouvait incapable de rendre impossible ce tout premier impensable comptable (l'inscription aux rôles de taillables fictifs). L'apaisement de ce conflit par la médiation royale en $1758{ }^{18}$ eut pour conséquence majeure d'assimiler le secours des sinistrés à l'action et à la bonne volonté de l'intendant, cantonnant ainsi les inquiétudes et les premières pratiques de recensement des officiers de la Chambre des comptes à une exigence de rigueur administrative théorique, mais non dépourvue d'effets. La collecte rigoureuse des renseignements nominatifs de chaque taillable sinistré qui était la condition sine qua none du dépôt d'une demande de dégrèvement en 1785 , si elle peut sembler insignifiante au vu des agissements des intendants successifs, retournait bien d'une toute nouvelle manière de penser le réel.

\section{Les fonds de secours spéciaux pour calamités agricoles en Isère (1860-1900)}

Dans la seconde moitié du XIXe siècle, l'état général de l'agriculture des pays méridionaux fut une préoccupation majeure pour l'élucidation des rouages mystérieux du progrès. La crise agricole de la seconde moitié du siècle entraîna une hausse de l'activité d'expertise sur ces territoires ${ }^{19}$. Il s'agissait alors de mettre en lumière les raisons du « retard » de l'agriculture dans les Alpes, au moyen des deux outils à la disposition de l'État: les recensements visant l'exhaustivité et portant sur quelques variables, et les monographies locales, restreintes spatialement mais faisant intervenir un plus grand nombre d'informations ${ }^{20}$.

Dans les conclusions de l'enquête agricole de 1866, les faiblesses de l'agriculture alpine sont présentées comme structurelles et même endémiques ${ }^{21}$. Marquée par un système de polyculture à forte tendance pastorale sur des terrains médiocres, accidentés et soumis aux intempéries, la production de ces départements de montagne était loin de rivaliser avec les régions céréalières du centre ouest ou les régions viticoles du sud. Pour ces experts, outre les affres de la mentalité routinière du paysan, l'absence d'investissement et la stagnation technique étaient à imputer directement à la carence du crédit agricole qui, par ailleurs, commençait à apparaître dans le bassin parisien et le Languedoc et restait intimement lié à la capa-

18. AD 38, 2C504, $\mathrm{n}^{\circ} 1$ : Lettre de Boullongue, secrétaire de D’Ormesson, à M. Bailly, président de la Chambre des comptes de Dauphiné à Grenoble, Versailles, 4 février 1758.

19. Pour un point sur la question, voir Louis-Antoine FABRE, La question agraire dans les montagnes francaises, Lyon, Publications de la Houille Blanche, 1914, 26 p.

20. Archives départementales des Hautes-Alpes (AD05), F2515: Allier, Rapport à M. le Préfet sur la situation de l'agriculture dans les Hautes-Alpes, ms., 1884, 88 p.

21. AD38, 139M20: Enquêtes sur la situation et les besoins de l'agriculture. Questionnaires et réponses des Commissions d'enquête (1866-1869). 
cité d'endettement que permettait les grands domaines ${ }^{22}$. Or, bien peu des 850.000 " exploitants de plus de 10 ha " que recense l'enquête agricole de 1892 résidaient dans les Alpes ${ }^{23}$. Dans ces régions de petites exploitations familiales, l'action publique visant à encourager et à encadrer l'agriculture recouvrait en gros cinq grands domaines: l'enseignement agricole ${ }^{24}$; la facilitation de l'enracinement des institutions agricoles ${ }^{25}$; une politique commerciale protectionniste; les lois de Restauration des terrains de montagne; diverses mesures fiscales comprenant notamment la diminution de la rente foncière et de la valeur vénale du sol, et l'attribution de secours et de dégrèvements fiscaux pour dommages environnementaux. Ainsi semblet-il possible de situer ces dernières aides comme une pièce à part entière dans ce que Gilles Postel-Vinay appelle la marqueterie de l'économie de mouvement à travers laquelle l'agriculture française du XIX siècle gagnerait à être étudiée 26.

À cette époque, les aides auxquelles pouvaient prétendre les cultivateurs alpins s'articulaient toutes sur les contributions directes ${ }^{27}$ et se divisaient en deux branches: les dégrèvements d'impôt et les fonds de secours spéciaux ${ }^{28}$. Depuis la loi de finances de 1819 , ces subsides étaient constitués annuellement par le produit d'un centime spécial additionnel au montant des contributions directes. Ces fonds étaient communs à tous les départements et concernaient « les habitants nécessiteux et non assurés, victimes de pertes résultant d'incendies, épizooties, orages, grêle, gelées, accidents divers, inondations et blessures ou morts accidentelles 29 ". À partir de 1851, le taux des secours devint uniforme pour tous les types de pertes et varia par la suite entre 4 et $6 \%$. Maurice Block rappelle à titre d'exemple que le crédit du centime spécial s'est élevé à 2.200.000 francs en 1875. Or, il souligne pour finir que la proportion de ce fonds était fixée « chaque année, par un arrêté ministériel, d'après le chiffre des ressources et d'après les dépenses indiquées par la moyenne des années antérieures 30 ".

À la grande différence du siècle précédent, les procédures de demande et de ventilation des fonds spéciaux pour calamités agricoles nécessitèrent de mettre en place un dispositif administratif nouveau capable de faire

22. Gilles Postel-VInAY, La terre et l'argent. L'agriculture et le crédit en France du XVIII au début du XXe siècle, Paris, Albin Michel, p. 281-354.

23. Ibidem, p. 284.

24. Création des professeurs départementaux d'agriculture par la loi du 16 juin 1879.

25. Notamment par le soutien aux comices et aux sociétés d'agriculture permettant la diffusion des concours agricoles.

26. Gilles POstel-VinaY, op. cit., p. 281.

27. Maurice BLOCK, "Dégrèvement " in Dictionnaire de l'administration française, Paris, BergerLevrault, vol. 1, 1877, p. 749.

28. Rappelons que les contributions directes se différenciaient des contributions indirectes, en étant perçu d'après un rôle nominatif des contribuables.

29. Maurice BLOCK, "Sinistre" in op. cit., vol. 3, p. 1658.

30. Ibidem, p. 1659. 
coexister dans le calcul de l'aide, deux conditions initiales contradictoires: l'indemnisation à hauteur d'une certaine proportion de toutes les pertes estimées par expertise et le plafond fixe établi par le gouvernement. La justice envers les cultivateurs nécessiteux était juridiquement garantie par la loi. Néanmoins, il incombait à la pratique administrative de rendre cette compensation possible sur un plan budgétaire. Pour ce faire, la procédure d'indemnisation des calamités agricoles retournait du travail parallèle de deux administrations principales: les services du préfet et l'administration des contributions directes.

À partir de 1880, les services préfectoraux furent chargés de l'élaboration des états annuels des sinistres ${ }^{31}$. Ces tableaux, proches en de nombreux points des recensements, étaient construits selon les découpages administratifs du territoire (cantons) et visaient principalement à établir les comptes exhaustifs de "perdants 32 " à l'échelle du département pour une année. Mais la nécessité dans laquelle se trouvait le préfet de fournir au ministère le chiffre des dépenses moyennes allouées au dispositif de secours pour son département, mena ces documents à prendre une teneur probabiliste nouvelle, perceptible dans le calcul des indemnisations moyennes propres à chaque type de sinistres ainsi que dans leur répartition mensuelle au sein de calendriers synchroniques ${ }^{33}$. Cette tendance évaluative/anticipative apparaît comme une nouveauté dans le sens où elle donne à voir l'action publique, aux prises avec le souci permanent d'une plus grande équité entre les citoyens, se mettre à agir " en moyenne » et à essayer de couvrir des risques par un commencement de péréquation statistique et assurantielle entre les individus ${ }^{34}$.

Mais l'aspect synoptique de ces états annuels de sinistres ne doit pas faire oublier que l'inscription de chiffres dans chacune de ces cases imprimées reposait sur l'effectuation d'une infinité de procédures d'ordre administratif. Toute demande de secours motivée devait invariablement suivre le même circuit. Lorsqu'une calamité frappait un ou plusieurs cultivateurs, les pertes devaient rapidement être estimées par le procès-verbal d'un officier de police judiciaire. Il s'avère que cette pièce originelle de la procédure revenait de manière générale aux brigades de gendarmerie ${ }^{35}$. Le principe

31. AD38, 93M1 : Sinistres. Statistique générale du département de l'Isère, annuel (1880-1905).

32. D'un point de vue administratif, la catégorie des " personnes ayant souffert d'un sinistre " compte les «perdants " (les sinistrés ayant perçu un secours); les « individus qui se sont pourvus en dégrèvement " (les sinistrés n'ayant pas perçu de secours et demandant un dégrèvement fiscal); et les individus n'ayant pas été reconnus comme sinistrés.

33. Ce glissement est visible en 1890, seule année pour laquelle on dispose de deux états des sinistres pour l'Isère: la version ancienne manuscrite relevant la liste différentielle des montants typologisés de perte et de secours par commune, et la nouvelle forme consistant en une série de " tableaux » imprimés à remplir par les différents rouages de la procédure.

34. Alain Desrosieres, Pour une sociologie historique de la quantification. L'argument statistique I, Paris, Presses de l'École des Mines, 2008, p. 110.

35. Parmi d'autres, AD38, 53M16: Procès verbal de gendarmerie constatant Dégâts occasionnés par une trombe d'eau et de la grêle aux propriétaires désignés ci-contre à la commune de Chantelouve. Pertes 
de ce document consistait à consigner par écrit la date de l'évènement, sa nature, la liste nominative des personnes touchées, leur commune de résidence, le montant estimatif des pertes individuelles et des pertes publiques, leur nature qualitative, si ces biens étaient assurés et auprès de quelle institution. Le procès-verbal était alors envoyé au procureur de la République et au préfet. Le préfet mandatait le contrôleur des contributions directes pour évaluer par expertise le montant des pertes. Ce chiffre était alors reporté à l'État annuel des sinistres en plusieurs temps.

D'abord, l'ensemble des dossiers dûment constitués devait passer par la direction générale des contributions directes sur laquelle le préfet n’avait aucune capacité d'influer et pouvait tout au plus soutenir l'une ou l'autre des plaintes qu'on lui avait adressées ${ }^{36}$. À ce stade, un ensemble de critères était vérifié, opération qui pouvait auquel cas automatiquement invalider un dossier. Le sinistré devait rapidement constituer sa demande après l'occurence du sinistre. Il devait avoir monté un dossier auprès de chaque commune sur le territoire desquelles il possédait des terres sinistrées. Enfin, il ne pouvait chercher à se constituer sinistré de plus d'une calamité par an. Pour tous les dossiers que le directeur des contributions directes refusait en raison de la contrainte du plafond annuel fixé par le gouvernement, le plaignant pouvait alors se pourvoir en dégrèvement, sachant que les montants obtenus par cette voie étaient infinitésimaux. Lorsque le dossier était accepté, la décision redescendait vers le maire, via le préfet. Une fois par an, les maires du département faisaient parvenir au préfet l'État annuel des sinistres de leur commune, que les services préfectoraux avaient ensuite pour mission de combiner en un seul État départemental.

Ainsi, les manières de calculer et de redistribuer des aides de l'État à la fin du XIXe siècle ne peuvent être entièrement déduites de la comparaison directe avec la situation telle qu'elle a été décrite au XVIII siècle. Deux éléments semblent avoir changé de nature. Si la collecte de renseignements auprès des potentiels ayants-droit à ces aides ponctuelles apparaît qualitativement similaire (liste exhaustive, informations nominatives), elle incombe désormais à un corps administratif non spécifique en terme d'agriculture, mais spécialisé dans la technologie d'écoute de l'esprit public propre aux sociétés disciplinaires: la gendarmerie. D'autre part, si ces deux situations permettent d'observer l'apparition d'une véritable législation visant à réduire les inégalités socio-spatiales, l'analyse montre que la recherche d'une plus grande justice dans la répartition des aides a été délégué toute entière à la rationalité administrative. Ainsi, l'État confia les

approximatives: 29150 frs, 26 aôut 1881; AD38 53M16: Procès verbal de gendarmerie constatant Dégâts occasionnés par des torrents aux propriétaires ci-contre de la commune d'Entraigues. Pertes approximatives: 1650 frs, 29 aôut 1881 .

36. Présente dans chaque département, l'administration des contributions directes dépendait directement du ministère des finances, voir Maurice BLOCK, "Contributions directes » in op. cit., p. 673. 
modalités d'application de ces procédures aux capacités d'improvisation d'un sens pratique administratif dont l'efficace devait découler d'une lutte de concurrence entre « services».

\section{Les indemnités de reconstitution agricole (1945-1960)}

Les violents combats qui jalonnent la Libération des Alpes françaises (notamment dans le Massif du Vercors, ou les hautes vallées du Queyras et de la Maurienne) entraînent des destructions considérables qui n’épargnent pas les villages de montagne et les fermes les plus isolées ${ }^{37}$. Malgré les rivalités initiales entre l'administration provisoire du Commissaire de la République, les services du ministère de la Reconstruction et de l'Urbanisme et les services du ministère de l'Agriculture, les différents responsables s'accordent sur le fait "qu'il ne s'agit pas de Reconstruire mais d'Édifier, [et] qu'il faut donc envisager un plan d'aménagement d'ensemble 38 ". Légitimée par la situation d'urgence, cette unanimité se fait en-dehors des délibérations du champ politique et passe plutôt par la mise en place de pratiques concrètes destinées à assurer plus qu'un retour à la normal pour les populations sinistrées: un nouveau départ.

Un arpentage minutieux du territoire permet de répertorier les destructions concernant les bâtiments et entreprises agricoles, les ateliers artisanaux, le cheptel vif et mort, les machines agricoles ${ }^{39}$. Dans le même temps, les enquêteurs projettent les principes qui devront guider la reconstruction: utilisation de matériel agricole en commun, politique de remembrement, mise à profit de la désorganisation du ramassage et du traitement du lait pour réaliser des installations équipées rationnellement et pour amener les agriculteurs à se grouper en coopérative, aménagements pour l'eau potable, mise en place de véritables « villages-coopératives " qui comprennent le travail de la terre et le traitement des récoltes et des produits de la ferme (laiteries et caves), regroupement de fermes isolées ${ }^{40}$. La reconstitution agricole apparaît clairement aux yeux des hauts fonctionnaires comme une occasion sans précédent de transformer la société paysanne alpine, de lui faire rattraper le " retard " pris sur les régions de grande culture, en somme de retrouver une certaine équité socio-spatiale à l'échelle du pays.

37. Pour l'inventaire des sites, voir: Luc BAZIN, Jean-Jacques LYON-CAEN, Jean-François LYON-CAENet Guy SCHNEEgANS, La reconstruction dans les Alpes françaises, 1945-1955, rapport de recherche BRA/ministère de l'Équipement, Laboratoire espace rural et montagne, École d'architecture de Grenoble, Grenoble, 1983, rapport non publié cité dans Jean-François LYON-CAEN, «Fermes du Vercors: ce qui change " in Regards croisés sur l'agriculture en Vercors. Hier, aujourd'hui, demain, ed. par Parc Naturel Régional du Vercors, 2003, p. 185

38. Archives nationales de France (ANF), F10/7103, M. BoIsSIERE, Activité à la tête du CARV, 17 novembre $1945,6 \mathrm{~F}$.

39. ANF, F10/7103: MM. CASAYS et THEVENOT, Compte-rendu de la mission effectuée dans le Vercors, 8 juin 1945, $26 \mathrm{f}$.

40. Ibidem. 
Participant aux travaux de la Commission parlementaire des Dommages de Guerre à l'été 1945, les fonctionnaires du ministère de l'Agriculture obtiennent une prise en compte spécifique des exploitations agricoles selon une triple exigence: sur le plan économique, porter au maximum le potentiel de production pour augmenter les ressources du ravitaillement; sur le plan social éviter l'exode, la disparition des fermes familiales et la concentration excessive des exploitations; sur le plan politique confirmer au paysan l'égalité des droits de tous les sinistrés. Le rapporteur ne se contente pas de rappeler que la reconstruction est orientée vers l'avenir, il défend également l'idée que « les règles de la reconstitution agricole permettent à l'agriculteur sinistré, voire même lui imposent, tous aménagements utiles à cette modernisation ${ }^{41}$ ".

Cette conception dirigiste s'appuie sur des aides très concrètes destinées à faciliter le remembrement, à améliorer les constructions rurales ${ }^{42}$, à moderniser les moyens de culture en conditionnant la participation financière pour moderniser l'équipement à l'agrément des services locaux de l'Agriculture, à proposer une indemnité d'éviction pour l'agriculteur à qui il serait impossible d'employer l'aide à la reconstitution de son exploitation. L'ensemble de ces subsides versés par l'État doit transiter par les Caisses de Crédit agricole qui s'occupent du versement et font bénéficier les agriculteurs de prêts à condition avantageuse, ce qui tend à renforcer la pénétration encore incomplète du crédit bancaire dans le monde paysan ${ }^{43}$.

Dans ce contexte, l'évaluation des destructions est un enjeu central : au niveau global, elle permet d'alimenter les revendications lors des négociations internationales; au niveau local, il s'agit de répartir les crédits au plus " juste ", c'est-à-dire en tenant compte pour chaque ferme non seulement des destructions mais aussi des projets de modernisation. Concrètement, cela se traduit par de multiples expertises dans les fermes endommagées. L'expert, nommé par le préfet sur proposition de la direction des Services Agricoles et en fonction de sa compétence spécifique à évaluer un domaine particulier (bâtiments, cultures, bétail...), doit effectuer des tournées communales ${ }^{44}$. Après que le sinistré a rempli un procès-verbal d'évaluation des destructions ayant affecté sa ferme, l'expert procède à la vérification de ces déclarations et transmet les procès-verbaux au Délégué départemental de la Reconstruction.

41. ANF, F10/7102: HoUDET, Rapport sur les dommages de guerre causés aux exploitations agricoles, 4 juillet $1945,15 \mathrm{f}$.

42. En laissant la possibilité au sinistré de cumuler le financement pour la reconstruction et la subvention du Génie rural pour l'amélioration de l'habitat.

43. Pour une histoire détaillée du développement du crédit agricole dans cette période, voir André GuesLin, Histoire des crédits agricoles, Tome 1: L'envol des Caisses Mutuelles (1910-1960), Paris, Economica, 1984, 955 p.

44. ANF, F10/7102: Commissariat Général aux Dommages de guerre du ministère de la Reconstruction et de l'Urbanisme, Instruction $n^{\circ} 13$. Expertises agricoles et artisanales rurales (Ordonnance $n^{\circ}$ 45-2063 du 8 septembre 1945), 24 janvier 1946, 40 p. 
Ensuite, la conversion des dégâts matériels en pertes comptables passe dans chaque département par une commission de fonctionnaires de la Préfecture et des Services agricoles qui établissent un barème précis des prix usuels ${ }^{45}$. Il doit définir: les prix de base (prix unitaire de tous les éléments d'exploitation, frais de repeuplement pour les cultures dont la durée de végétation est supérieure à un an, coût de la reconstitution physique ou chimique des sols), les rendements (poids moyen de certains animaux, rendement moyen de certaines cultures), les forfaits pour certaines cultures légumières, les quantités maxima d'approvisionnement au cours d'une campagne (en fonction des quantités d'engrais disponibles par exemple). Le ministère fournit des tableaux récapitulatifs qui matérialisent ce découpage et imposent les critères de définition de chaque produit. Cet agencement est repris à tous les échelons administratifs depuis les services des ministères jusqu'aux experts locaux et même, ou plutôt surtout, jusqu'aux sinistrés eux-mêmes.

Cette procédure d'évaluation débouche sur l'attribution de réparations (fiduciaires) après examen des dossiers et établissement d'un ordre de priorité qui tienne compte de l'importance des destructions et de la précarité de la situation du sinistré et de sa famille ${ }^{46}$. C'est donc uniquement à l'intérieur de ce dispositif complexe d'enquête-évaluation-conversion que les sinistrés peuvent saisir (au sens juridique) l'État pour demander réparation et réciproquement, c'est par le même biais que l'administration peut saisir (connaître) ses administrés sinistrés. Ce processus d'assujettissement se noue à la croisée de deux légitimités différentes: les sinistrés sont en attente d'une indemnisation au plus près possible des pertes qu'ils ont subi alors que les services de l'État sont dans la perspective d'une réorientation des activités agricoles qui nécessite un quadrillage très serré du monde rural. Et il se construit principalement à partir des techniques du recensement et de l'enquête.

Au final, l'activité règlementaire et pratique de l'administration a supplanté le débat parlementaire mais a dû composer avec les réticences des sinistrés à épouser la logique du progrès. La subvention agricole a le plus souvent pris la forme de l'indemnisation, passant par l'attribution de subsides, de prêts bonifiés et l'exécution de travaux directement sous le contrôle de l'État. Elle a nécessité un travail d'enquête extrêmement précis qui est resté dans les cartons de l'administration et n'a pas bénéficié d'une véritable publicité, élément important qui va évoluer par la suite. La

45. ANF, F10/7102: Commissariat Général aux Dommages de guerre du ministère de la Reconstruction et de l'Urbanisme, Instruction technique sur l'établissement des barèmes agricoles, 9 avril $1947,17 \mathrm{f}$.

46. Dans le cas d'une destruction totale, les travaux peuvent être pris en charge directement par les services de l'État qui sous-traitent la réalisation des plans et des travaux. Voir par exemple le fonds de l'architecte André Demire aux Archives Départementales de l'Isère (155J). 
rationalité administrative ne s'est pas véritablement imposée et les opérations de reconstitution agricole restent marquées par le sceau du compromis. Elles n'autorisent pas un retour à la situation antérieure pour les paysans mais elles n'anticipent pas pour autant totalement les recommandations de l'agriculture productiviste.

\section{Les subventions pour la modernisation des zones-témoin (1952-1960)}

L'opération dite des "zones-témoin " est issue d'une initiative de l'AGPB (Association Générale des Producteurs de Blé) relayée par les crédits de l'État. Concrètement, cela passe d'abord par la création en janvier 1947 du Fond National de Progrès Agricole, financé à la fois par l'AGPB et par l'État. Chaque année, le Fond établit un programme qui associe mise au point de techniques nouvelles et vulgarisation. L'AGPB prend alors en charge l'exécution de ce programme après avoir reçu l'aval du ministère. Les grands céréaliers sont là dans une stratégie de défense de leur propre position sur le moyen terme, qui nécessite leur implication directe dans de nouvelles formes de vulgarisation: une agriculture modernisée et bien organisée permettrait à la France de se positionner comme une puissance agricole européenne et mondiale ${ }^{47}$.

À partir du début des années 1950, ces opérations innovantes vont reconfigurer de façon décisive les liens entre subvention et collecte de renseignements, en radicalisant les lignes entrevues lors du moment de la reconstitution agricole: on tend à sortir de la logique de l'indemnisation pour privilégier celle de la réorientation, ce qui ne veut pas dire que la première disparaisse mais simplement que sa légitimité propre est de moins en moins évidente. La reconnaissance de l'inégalité socio-spatiale donnant lieu à une subvention publique est conditionnée par le fait que les paysans acceptent de nouvelles pratiques à même de résorber l'inégalité en cause. En effet, les zones-témoins ont désormais pour objectif de lier l'aide matérielle et financière effective avec des conseils techniques précis 48 .

Ensuite, les nouvelles opérations de vulgarisation ne prennent plus en charge une parcelle, un champ ou une ferme mais une véritable portion de territoire, d'abord un village (les premiers villages-témoins), puis des zones moins définies, les zones-témoins qui peuvent s'étendre sur une surface proche de celle d'un canton sans pour autant en respecter les limites administratives. Ce changement d'échelle se fait dans un souci d'efficacité. Il s'agit d'obtenir des résultats rapides et spectaculaires en terme d'augmen-

47. AD38, 3426W211: Groupement de productivité agricole du Trièves, Le paysan du Trièves dans la voie du progrès. Bulletin des adhérents de la zone-témoin du Trièves, $\mathrm{n}^{\circ} 2$, octobre $1952,19 \mathrm{f}$. 48. Ibidem. 
tation de rendements sur des communes « choisies pour être bien démonstratives " (rendements faibles et niveau d'équipement limité ${ }^{49}$ ) ou des zones peu modernisées et présentant une certaine cohérence géographique (en Isère, les commentaires sur la configuration du Trièves en " cuvette " alimente les rapports de nombreux observateurs ${ }^{50}$ ).

Enfin, l'Association Générale des Producteurs de Blé a prévu de déléguer un agent technique pour superviser au quotidien chaque villagetémoin ${ }^{51}$. Cette fonction est rapidement confiée pour les zones témoins à un salarié de la Chambre d'agriculture, qui est d'ailleurs souvent le premier salarié de cette institution en pleine renaissance ${ }^{52}$. Le rôle de ce technicien est pensé dès le départ comme le pivot de toute l'entreprise. Sa présence constante doit lui permettre à la fois d'inciter-encourager-conseiller et de surveiller-orienter-contrôler. Il est l'intermédiaire, le traducteur, la « cheville ouvrière ", comme ne cessent de le répéter les sources et les acteurs eux-mêmes ${ }^{53}$. Interlocuteur privilégié à la fois des agriculteurs de la zone et des responsables agricoles départementaux administratifs ou professionnels, il se charge de faire appliquer le programme mis au point pour chaque opération témoin.

Une fois l'emplacement et les limites de la zone-témoin validés par la Chambre d'agriculture et les Services Agricoles du département, le conseiller agricole doit commencer son travail par une enquête précise sur la situation économique et sociale des paysans qui adhèrent au Groupement de Productivité Agricole ${ }^{54}$. Au cours de ses visites individuelles, il s'agit pour le conseiller de convaincre afin d'obtenir l'accord de l'exploitant pour un programme de quatre années répondant aux besoins précis de l'exploitation analysée ${ }^{55}$. Il est prévu que "l'action des techniciens s'exerce suivant des méthodes comparables et que, d'autre part, les programmes individuels d'action se traduisent par des résultats prévisionnels, chiffres également comparables entre eux. Pour satisfaire cette double condition, il a été établi un plan-type d'analyse d'une exploitation agricole qui fait apparaître en volume et en valeur les incidences du programme

49. Ibid.

50. Voir par exemple: AD38, 3426W210: direction des Services Agricoles de l'Isère, Rapport sur la physionomie, les vocations et l'avenir du Trièves, 1952.

51. AD38, 3426W212: Commission du Blé du département de l'Isère, Compte-rendu de réunion pour le choix du village-témoin, 11 août 1951, $3 \mathrm{f}$.

52. AD38, 139M62: Chambre d'agriculture de l'Isère, Procès-verbal de la session ordinaire du 4 novembre 1952 de la Chambre d'agriculture de l'Isère.

53. Fonds personnel, entretien de l'auteur avec un conseiller agricole devenu Directeur de la Chambre d'agriculture de son département, 7 août 2009.

54. Chaque zone-témoin nécessite la création d'un Groupement de Productivité Agricole: les paysans adhérents à cette association suivent le programme défini par le conseiller et se répartissent les financements.

55. AD38, M3947: direction de la Production Agricole du ministère de l'Agriculture, Instruction relative aux modalités d'application de l'aide technique et financière de productivité, $10 \mathrm{p}$. 
d'aide technique et financière ${ }^{56}$ ». Ce travail de collecte des informations permet de comptabiliser et mesurer des productions quantifiables mais un second aspect est peut-être encore plus capital: le plan-type d'analyse permet de convertir les différentes pratiques et leurs impacts en données chiffrées, de les aborder ainsi sous l'angle de la rationalité économique en permettant une comparaison entre différentes années sur une même exploitation et également entre différentes exploitations. En fonction des résultats obtenus, le conseiller est à même de dire les bonnes et les mauvaises pratiques sans jamais se départir de sa légitimité comptable.

C'est à ce niveau qu'on peut mesurer véritablement le poids des subventions agricoles dans ce dispositif. La première année de fonctionnement de la zone-témoin, les taux de subvention atteignent $15 \%$ des investissements totaux (10\% par la suite) qui doivent comporter au moins $20 \%$ (puis $30 \%$ ) d'autofinancement de la part des adhérents. L'essentiel des investissements passe alors par la mise en place de prêts bonifiés avec l'aide de l'État. Sur le plan financier, l'agriculteur s'engage auprès du groupement qui s'engage lui-même vis-à-vis de la Caisse Nationale de Crédit Agricole pour obtenir les prêts à caractéristiques spéciales ${ }^{57}$. Sur le plan administratif, des procédures permettent de faciliter l'attribution des subventions et la réalisation rapide des prêts grâce à l'assouplissement des garanties demandées par la Caisse Régionale de Crédit Agricole 58. Ce montage financier est destiné à assurer l'adhésion des agriculteurs au processus de modernisation. Il n'est plus question qu'ils puissent bénéficier d'aides s'ils n'investissent pas leurs fonds propres et s'ils ne se conforment pas au programme de développement.

Dans la pratique, les desseins des hauts fonctionnaires du ministère de l'agriculture ne sont pas entièrement respectés. Après quelques mois de fonctionnement, un rapport montre que si les subventions atteignent les montants prévus, le rapport entre le montant des crédits de subventions et des crédits de prêts ouverts pour chaque zone-témoin n'est que très rarement respecté 59 . Et de même que les paysans ne se conforment pas entièrement aux plans qui leur sont proposés, les conseillers agricoles ne sont pas les simples courroies de transmission des directives administratives. Si les fiches qu'ils remplissent leur permettent de dresser un classement des adhérents selon une logique comptable, leurs visites ne se réduisent pas à cette dimension. En acquérant une connaissance intime des fermes qu'il

\footnotetext{
56. Ibidem.

57. Ibid. Prêts à caractéristiques spéciales: $3 \%$ sur 10 ans : acquisition de cheptel mort ou vif par un syndicat, une coopérative ou en copropriété, travaux fonciers; $4 \%$ sur 4 ans pour: engrais, désherbants, amendements, semences, plants, travaux de désinfection ou de défonçage.

58. Ibid.

59. AD38, M3947: direction de la Production Agricole du ministère de l'Agriculture, Instruction Z.T. 3, 10 p.
} 
visite et des familles qui les exploitent, le conseiller établit un classement pratique des pratiques qu'il recense en fonction de ses propres présupposés. Sans pouvoir développer ici la description de cette position complexe, on peut remarquer deux choses. Ce contact introduit a priori un biais humain dans la logique administrative des enquêtes et de la juste répartition des crédits qui doit s'en suivre au risque d'en diminuer l'efficacité. Mais en réalité, tout se passe comme si cette capacité de contact et d'encadrement au plus près des pratiques réelles des paysans permettait une efficacité accrue du dispositif d'incitation à la modernisation. Enquêté, subventionné, conseillé, endetté auprès du Crédit agricole, le paysan de la zone-témoin est conduit à intérioriser progressivement lui-même la rationalité économico-administrative qui pouvait lui apparaître plus extérieure au moment des opérations de reconstitution agricole.

Au final, la description et l'analyse de ces quatre moments dans l'histoire de la subvention agricole offre la possibilité d'une comparaison combinatoire qui ne se limite pas à la construction d'un récit chronologiquement ordonné. En apparence, tout se passe comme si, pendant plus de deux siècles et demi, le pouvoir persistait à maintenir l'équité entre les territoires qu'il contrôle en utilisant notamment la subvention agricole. Pourtant, l'observation des différentes configurations des échanges d'aides et d'informations entre l'État et le monde paysan alpin permet d'élucider la nature ambivalente du principe de justice lorsqu'il s'applique aux sociétés rurales à travers les différents dispositifs de subvention.

Dans cette optique, chaque moment doit être examiné relativement aux trois autres. Les dégrèvements extraordinaires de taille au XVIII siècle mettent en lumière une emprise administrative assez faible de la Chambre des comptes sur un territoire peu homogène simplement unifié par une même fiscalité (pays d'élection, généralités) : elle se limite à informer le souverain des agissements de l'Intendant auquel revient la demande et la distribution des secours après sinistre. Les fonds spéciaux du XIXe siècle révèlent l'efficacité croissante de procédures administratives uniformisées et adaptées au nouveau maillage du territoire (départements, cantons) : les services préfectoraux agissent sous le contrôle de l'administration des contributions directes dans le cadre d'une politique de compensation financière nécessitant l'évaluation des montants des dégâts et la ventilation d'aides ponctuelles. Les indemnisations de reconstitution agricole après la Seconde Guerre Mondiale découvrent la capacité des services des ministères de l'Agriculture et de la Reconstruction à descendre au niveau des fermes endommagées dans chaque commune: dans ce contexte d'urgence nationale, leur comptabilité des destructions les placent en position de force pour la construction légitime d'un projet de modernisation qui passe par 
le double dépassement des réticences paysannes et des méandres du débat politique parlementaire. Dans les années 1950, les subventions pour la modernisation des zones-témoin dévoilent un désengagement des services de l'État proprement dit, qui confient leur pouvoir aux organisations professionnelles agricoles en créant des territoires spécifiquement dessinés par les circuits de répartition des crédits publics: les conseillers agricoles travaillent au sein de ce dispositif d'incitation permanente qui tend à favoriser l'intériorisation de la logique administrative par les paysans eux-mêmes.

Les différentes lignes tracées ici permettent à présent d'élucider le rapport entre les deux exigences de justice qui sous-tendent cette archéologie de la subvention agricole, pour mémoire la décision politique d'une compensation des inégalités socio-spatiales et le travail administratif d'une plus juste répartition des aides. Plutôt que de penser l'éclipse du premier terme derrière le second, il s'agirait de décrire comment le dispositif de subvention permet «la production de passage ou de porosité entre ces sphères auparavant distinctes, c'est-à-dire la production de visibilité ou de transparence 60 ». Dans un premier temps, la juste répartition des aides s'inscrit progressivement dans la loi, permettant de légitimer un contrôle de l'administration sur les décisions d'indemnisations ponctuelles. Le répartir juste consiste alors à compenser les pertes des ayants-droit. Dans un second temps, cette notion se retrouve au cœur du projet politique de modernisation et tend à être intériorisée par les paysans individuellement subventionnés et orientés. Le répartir juste revient cette fois à les inciter à se conformer à la norme du progrès. Par la circulation de renseignements et d'argent qu'elle implique, la subvention n'apparaît plus seulement comme un outil de politique publique, mais aussi comme ce dispositif producteur de visibilités dans lequel le réel recensé devient le seul réel qui compte. À travers cette archéologie de la subvention, les inégalités socio-spatiales justifient successivement une indemnisation-compensation travaillée par une lutte pour la construction du droit et une subvention-incitation sous-tendue par une production de la norme, permettant de " contrôler tout en ne faisant rien, c'est-à-dire en prétendant ne rien faire d'autre que de montrer les choses telles qu'elles sont ${ }^{61}$ ». C'est la construction sociale-historique de cette morale du réel qui apparaît finalement au cœur des rapports de l'enquête et de la subvention.

60. Thomas BERNS, Gouverner sans gouverner. Une archéologie de la statistique, Paris, PUF, 2009, p. 137. 61. Ibidem. 